\title{
Role of Stem Cell Therapy in Diabetic Cardiomyopathy in Rats: Histological and Immunohistochemical study
}

Hanan Dawood Yassal, Abdalwakel Alsayed Mohammed2, Azza Saleh Moawad3, Ahmed Yahia Sedeakl, Gaber Hassan Abdelfatah1.

1: Anatomy and Embryology department, Faculty of Medicine, Beni-Suef University, Egypt

2: Anatomy and Embryology department, Faculty of Medicine, Cairo University, Egypt

3: Histology department, Faculty of Medicine, Beni-Suef University, Egypt

\begin{abstract}
:
Background: Diabetes mellitus is one of the main causes of death due to the complications that involve many organs such as heart, kidney, retina and others. Mesenchymal stem cell (MSC) has been demonstrated to be effective in treatment of diabetes. Aim of the work: To evaluate the effect of stem cells in the treatment of diabetic cardiomyopathy clarifying their role in oxidative stress and inflammation. Material and methods: 30 adult male albino rats were equally divided into 3 groups: control group (group I): received $1 \mathrm{ml}$ saline by intra-peritoneal (IP) injection. Streptozotocin-treated group (group II): received Streptozotocin (STZ) $(60 \mathrm{mg} / \mathrm{kg}$ BW, I.P.) for induction of diabetes then were sacrificed after 4 weeks. Steptozotocin+ Stem cell-treated group (group III): received STZ for induction of diabetes and they were left for 4 weeks then they were injected once intravenously with 1 million units of MSC then were sacrificed after 4 weeks. The heart sections were examined Histologically with H\&E and Masson's Trichrome Stain and Immunohistochemically for endothelial Nitric Oxide Synthase (eNOS). A morphometric study and statistical analysis were performed. Results: DM caused inflammation, degeneration, fibrosis and decreased eNOS Immunoexpression of the cardiac muscle fibers. The administration of MSCs improved pathological changes and increased eNOS Immunoexpression in the heart. Conclusion: MSCs administration proved to have effective therapeutic role in treating diabetic cardiomyopathy.
\end{abstract}

Keywords : Diabetes, Cardiomyopathy, Mesenchymal stem cell, eNOS, Oxidative stress. 


\section{Introduction:}

Diabetes mellitus (DM) is considered one of the widest spread non communicable metabolic diseases. Recently, there is a sudden increase of occurrence of diabetes all over the world. The International Diabetes Federation (IDF) denoted that the number of diabetic patients will increase from 415 million in 2015 to 642 million by 2040 [1]. Diabetes causes lot of complications involving microvascular complications that include retinopathy, nephropathy and neuropathy; and macrovascular complications such as cardiomyopathy, hypertension and peripheral arterial disease [2].

Diabetic cardiomyopathy is a macrovascular complication in diabetic patients and it is considered one of the main causes of morbidity and mortality in those diabetic patients [3]. Streptozotocin (STZ) is a type of antibiotics that can be obtained from Streptomyces achromogenes. It has been used on a large scale for producing experimental diabetes mellitus in many animals as it induces degeneration of pancreatic $\beta$ cells causing deficiency of insulin secretion [4].
Treatment using Stem cells is a modern type of intervention strategy that is introduced into the injured organs and tissues to treat diseases such as diabetes mellitus and its complications. Many researchers consider that stem cells are able to take a role in the treatment of human diseases and relieve suffering. The stem cells possess the ability of proliferation and giving rise to subsequent generations with multiple degrees of differentiation capacities. This ability exhibits important potential for the generation of tissues that can substitute diseased and damaged tissues in the body [5].

Mesenchymal stem cells (MSCs) are characterized by having properties such as antioxidant and anti-inflammatory effects enabling them to be used in decreasing tissue necrosis. $[6,7]$ The goal of this study was to reveal the changes induced by diabetes on the heart clarifying the possible therapeutic effects of MSCs through histological, immunohistochemical and biochemical studies. 


\section{2- Material and Methods:}

The study was carried out at the Animal House of Faculty of Medicine, Cairo University, according to the guide lines used for the care and the use of laboratory animals (code of ethical approval is 018-61).

I-Experimental Animals: The study was performed on 30 adult male albino rats of an average weight $150-180 \mathrm{~g}$. The rats were acclimatized in the laboratory for a period of two weeks before performing the experiment. They were manipulated in cages made of metal under standard laboratory and pathogen-free environmental conditions. The rats were nourished using standard levels of the rodent water and food. These rats were equally divided into 3 groups: Control group (group I): this group received $1 \mathrm{ml}$ saline by intra-peritoneal (IP) injection. Streptozotocin-treated group (group II): this group received Streptozotocin (STZ) $(60 \mathrm{mg} / \mathrm{kg}$ body weight (BW), I.P.) for induction of diabetes then sacrificed after 4 weeks [8]. Steptozotocin+ Stem cell-treated group (group III): this group received STZ for induction of diabetes and left for 4 weeks then these rats were intravenously injected with 1 million units of bone marrow-derived stem cell (BMDSCs) only for one time. After 4 weeks of being treated with stem cells, these rats were sacrificed [9].

II- Chemicals: 1-Streptozotocin (STZ): was obtained from Sigma Company (St. Louis Mo, USA) in the form of powder solvent. Each vial of Streptozotocin powder contain 1 gram of Streptozotocin active ingredient with the chemical name, N(Methylnitrosocarbamoyl)- $\alpha-$ D-glucosamine Streptozotocin.

2-Fluorescent bone marrow-derived mesenchymal stem cells(BM-derived MSCs): The preparation of the stem cells was carried out in the stem cell unit, Biochemistry department, Faculty of Medicine, Cairo University.

Induction of DM: STZ $(60 \mathrm{mg} / \mathrm{kg} \mathrm{BW})$ was dissolved in $0.1 \mathrm{M}$ sodium citrate buffer in Biochemistry department, Faculty of MedicineCairo University. Preparation of the solution was done at $\mathrm{pH} \quad 4.5$ and then injected intravenouslywithin $15 \mathrm{~min}$. The aim of this procedure is to induce T1DM [10]. 
Diagnosis of Diabetes: Polydipsia and Polyphagia were observed in adult rats within three days of injection ofstreptozotocin and this suggested DM due to destruction of $\beta$-cells of Langerhans islet cells [11]. Diagnosis of diabetes was approved by elevation of blood glucose level [12].Rats with blood glucose levels more than $200 \mathrm{mg} / \mathrm{dL}$ were considered diabetic [10].

Treatment of DM: Preparation of Labeled bone marrow-derived mesenchymal stem cells was done in the stem cell unit, Biochemistry department, Faculty of Medicine, Cairo University. These stem cells were given by single I.V. injection in a dose of $1 \mathrm{ml}$ of about $\left(1 \times 10^{6}\right.$ cells/rat $) 4$ week after confirmation of diabetes [13]. The treatment was allowed only to the third group suspended in $1 \mathrm{ml}$ normal saline [14].

Isolation and culture of bone marrowderivedmesenchymal stem cells(BM-derived MSCs)[15]: 5-bromo-2'-deoxy-uridine (BrdU) labeled MSCs were prepared at Medical
Biochemistry Department, Faculty of medicine, Cairo University.

Isolated and cultivation of BM-derived MSCs were carried out for 4 weeks. Adult rats were euthanized and bilateral femora and tibias were removed under sterile conditions and placed in Dulbecco's modified eagle medium (DMEM; Gibco/BRL). Flushing out of MSCs was performed with DMEM using a syringe fitted with a 23-guage needle. This was followed by gently pipetting bone marrow from each bone many times to separate cells. Then the cells were washed two times using DMEM, centrifuged $2250 \mathrm{rpm}$ for $15 \mathrm{~min}$, and cultured in DMEM supplemented with $10 \%$ fetal bovine serum (GibcoBRL), $100 \mathrm{U} / \mathrm{ml}$ penicillin $\mathrm{G}$ and 100mg/ml streptomycin (GibcoBRL) at $2.5 \times 10^{5} / \mathrm{cm}^{2}$. The cells were incubated at $37{ }^{\circ} \mathrm{C}$ in humidified $95 \%$ air and 5\% CO2. 3 days later, elimination of non-adherent cells was done. Addition of fresh complete culture medium DMEM was done and then replaced every 3 or 4 days. When the cells become $80-90 \%$ confluent over 14 days, they were harvested with $0.25 \%$ trypsin and one mmol EDTA (GibcoBRL) for 3 
min at $37^{\circ} \mathrm{C}$, replanted in six-well disk at $1.5 \times 10^{5} / \mathrm{cm}^{2}$ and again grown to near confluence. Dilution of cells was obtained by adding water 1:2 per passage to expand the culture.

Fluorescence phase-contrast microscope (Axiocam MR R3, Carl Zeiss, Germany) was used to observe the rats MSCs every 2 or 3 days. Histological study: Anaesthesia of The rats was performed using mild ether inhalation then subsequent sacrifice of these rats was done by cervical dislocation to avoid chemical injury [16].

Each rat's heart was excised carefully immediately. The hearts were dissected, fixed in $10 \%$ formalin overnight, processed for paraffin blocks and sectioned at $5 \mu \mathrm{m}$ thickness.

Sections of paraffin were used in: 1Hematoxylin \& Eosin stain [17]. 2Masson'strichrome stain [18]:to detect collagen fibers deposition. 3-Immunohistochemical study using endothelial nitric oxide synthase (eNOS)
[19]: Primary antibody: endothelial Nitric Oxide Synthase (eNOS) antibody:

It is Mouse monoclonal [M221] antibody (igG1) (abcam Medical, Cambridge, USA, catalogue number ab76198) to eNOSand it consists of Recombinant part of mouse eNOS protein which contained residues of amino acid in the $\mathrm{C}$ terminal region. It is liquid and stored at $-20^{\circ} \mathrm{C}$ in $0.05 \%$ Sodium Azide. Kidney section was used as a standard positive control. One of the heart sections was used as a negative control by avoiding application of the primary antibody.

Fluorescent microscopic examination: Heart Sections of STZ+stem cell group were submitted to fluorescent microscopy examination to clarify fluorescent labeled mesenchymal stem cells (Fig. 1). It was done by detecting the Bromodeoxyuridine (BrdU) - positive cells in the sections. The sections were immunostained using mouse anti- BrdU (1:100, Neomarkers), and goat anti-mouse Ig GFITC (1:100, Kpl). 


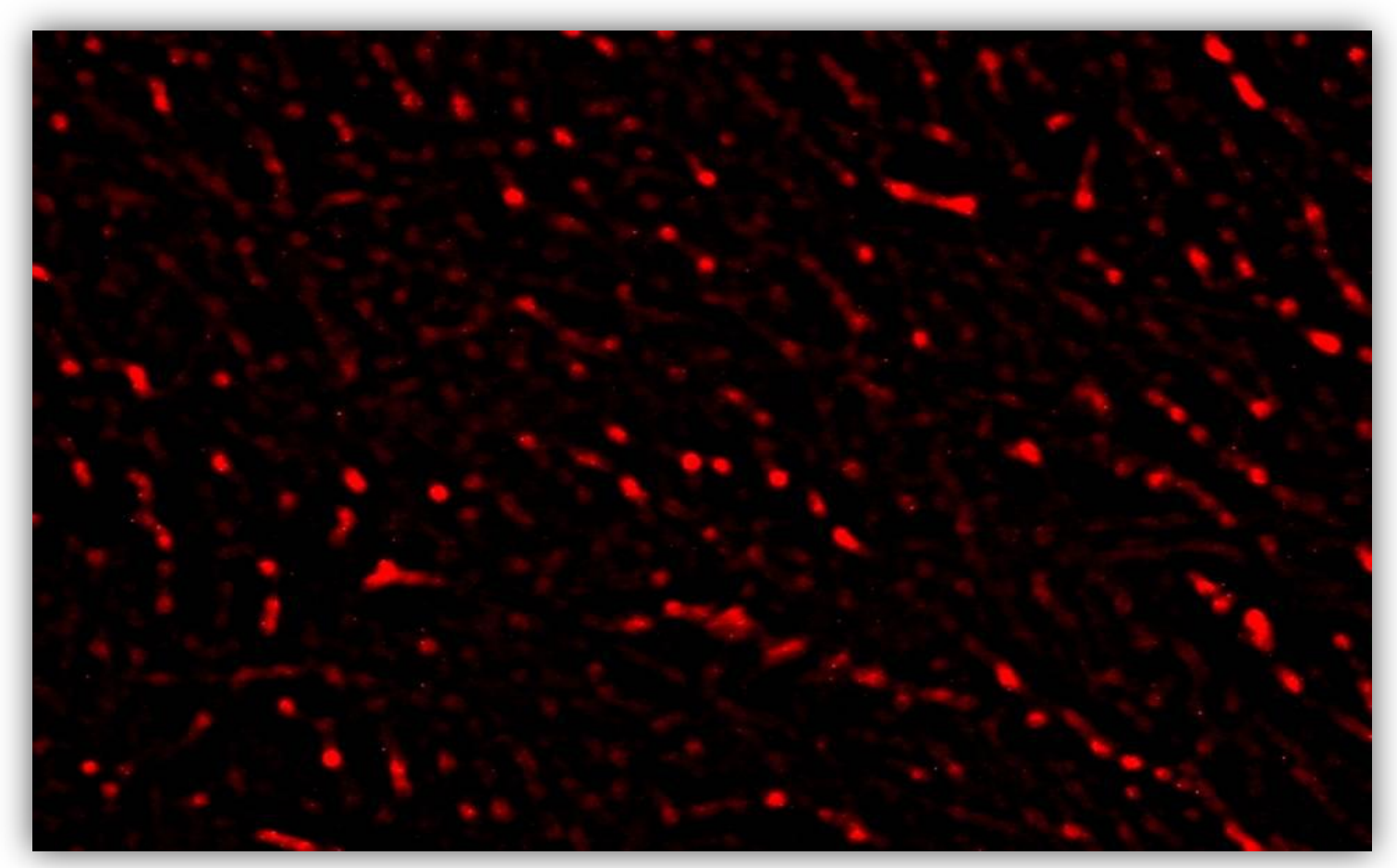

Fig. (1): A micrograph from fluorescent microscope of a heart section of the STZ+MSCs group showing fluorescent illumination of fluorescent-labeled mesenchymal stem cells. (Immunostaining; X 400)

Morphometric study: Using a Leica Quin 500 (Leica Ltd, Cambridge, UK) between rat groups. The results of Statistics computerized image analysis system, the morphometric studies were done for the mean area of the collagen fibers in the heart sections and the intensity of the eNOSimmunoexpression in the heart sections.

\section{Statistical analysis [20]: Analysis of}

Statistics was done using SPSS software, version 16. All data will be expressed as mean \pm SD. One way analysis of variance
(ANOVA) test will be used for comparison were considered significant when the p-values were $<0.05$.

\section{3- Results:}

No deaths were detected in all rats.

\section{$\underline{H \text { and } E \text { stain results ( fig.2,3): }}$}

In the control group, $\mathrm{H}$ and $\mathrm{E}$ stained sections of the hearts showed normal cardiac muscle architecture in the form of longitudinal 
striated and branched fibers having central oval nuclei and acidophilic sarcoplasm (figs.2A\&3A). Also, normal blood capillaries were demonstrated (figs.3A).

In the STZ-treated group, the sections of the hearts revealed disorganization and separation of the muscle fibers with blood extravasation between muscle fibers (figs.2B\&3B).There were degeneration and loss of the striations of the muscle fibers with small dark (pyknotic)

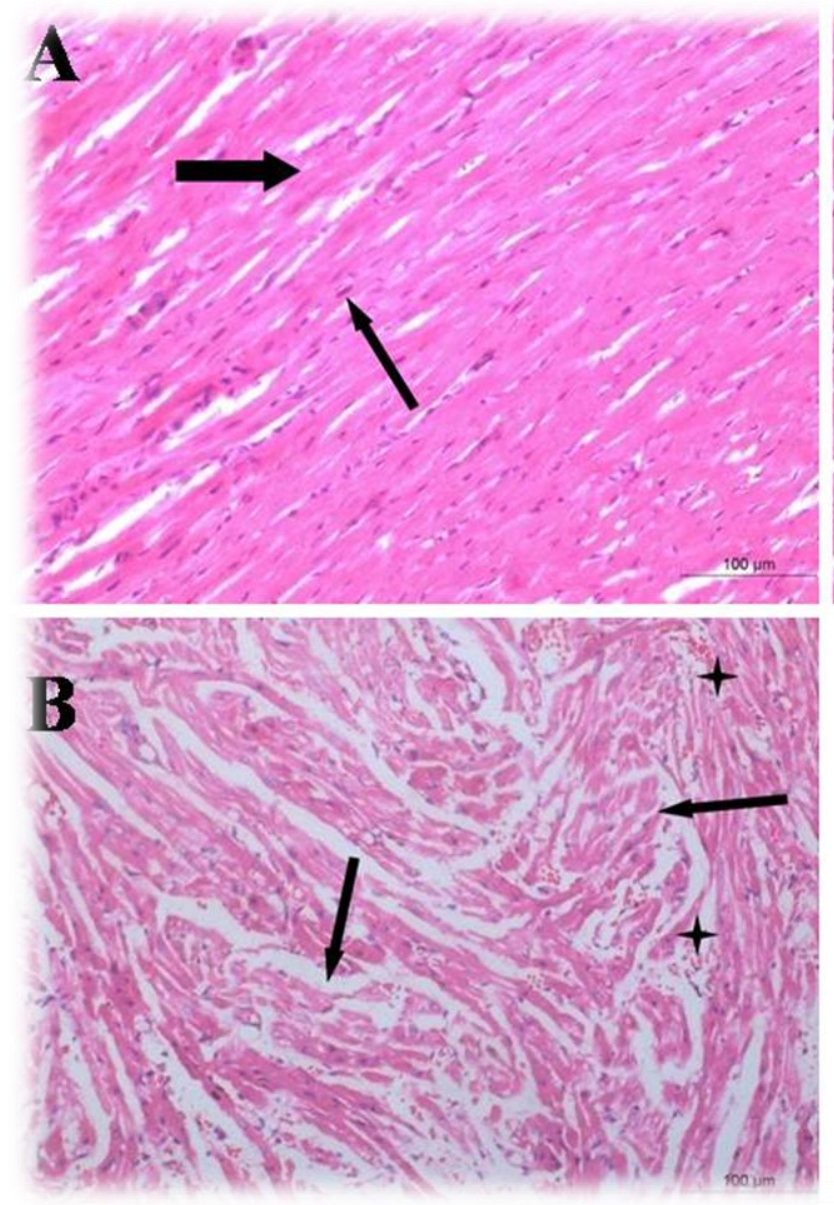

nuclei and cytoplasmic vacuolations (figs.3B) and dense mononuclear cellular infiltration (fig.3C).

The hearts of STZ+ Stem cell-treated group revealed nearly normal muscle fibers with few separated fibers (figs.2C\&3D). Little mononuclear cellular infiltration was also noticed (fig.3E).

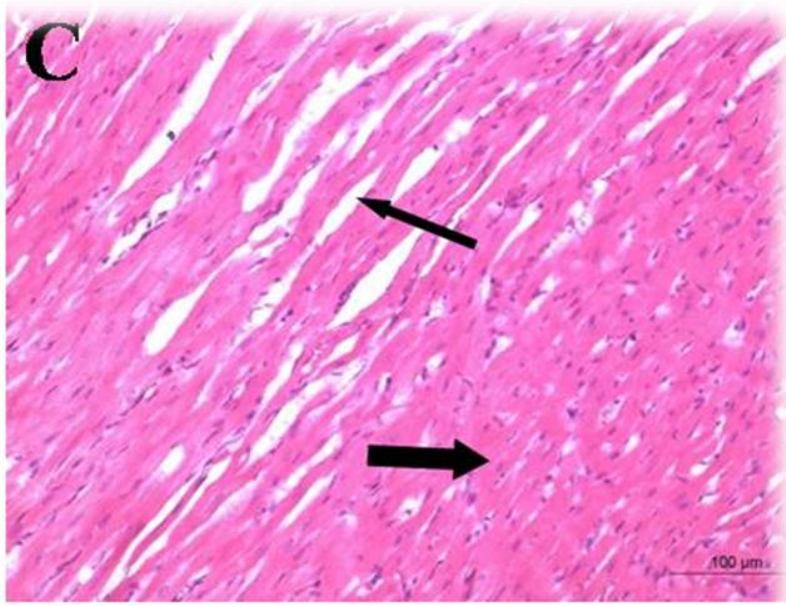


Fig. (2): A photomicrograph of $H \& E$ stained heart sections of control (A), STZ treated (B) and STZ+ stem cell treated $(C)$ groups. (A) showing normal cardiac muscle in the form of longitudinal striated and branched muscle fibers (thick arrow) having acidophilic sarcoplasm with central oval nuclei (thin arrow). (B) showing disorganization and separation of the fibers (arrows) and blood extravasation (stars). (C) showing nearly normal muscle fibers (thick arrow) with few separated fibers (thin arrow). (H \& $\boldsymbol{E}, \boldsymbol{x 2 0 0})$
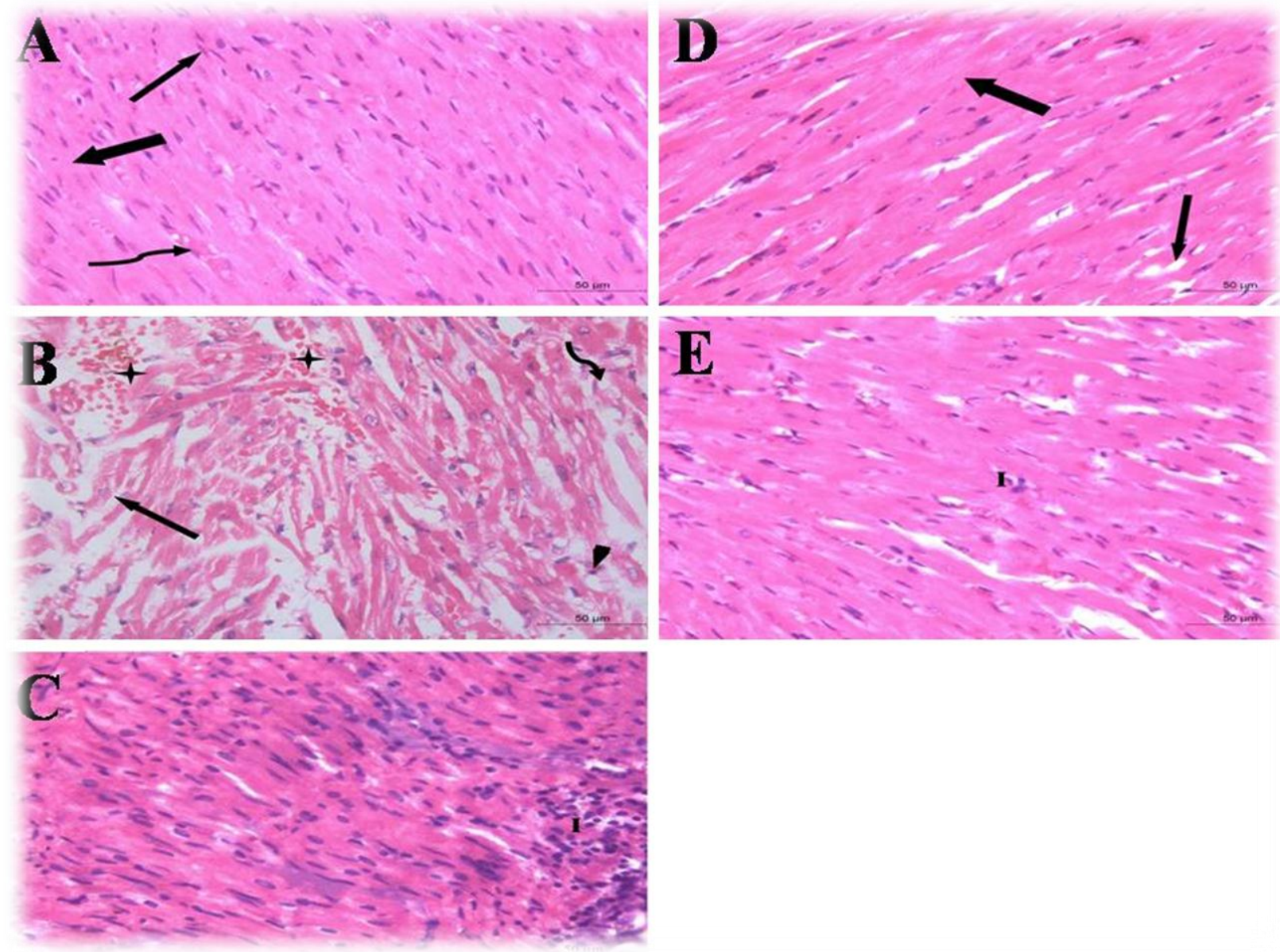

Fig. (3): A photomicrograph of higher magnification of $H \& E$ stained heart sections of control $(A)$, STZ treated $(B, C)$ and $S T Z+$ stem cell treated $(D, E)$ groups. $(A)$ displaying normal cardiac muscle in the form of longitudinal striated and branched muscle fibers (thick arrow) with central oval nuclei, acidophilic sarcoplasm (thin arrow) and blood capillaries (curved arrows). (B) displaying degeneration, loss of the striations and separation (arrows) of the fibers with small dark nuclei (arrow head), cytoplasmic vacuolations ( curved arrow) and blood extravasation (stars). (C) displaying dense 
mononuclear cellular infiltration (I). (D) displaying apparently normal muscle fibers (thick arrow) with few separated fibers (thin arrow). (E) showing little mononuclear cellular infiltration (I). (H \& $\boldsymbol{E}, \boldsymbol{x} 400)$

\section{Masson's trichrome stain results (figs. 4):}

Examination of Masson's trichrome stain revealed that the control hearts showed muscle fibers with minimal collagen fibers deposition (figs.4A).However, in STZ-treated group,the hearts were displaying muscle fibers and capillaries with massive collagen fibers deposition (figs.4B). While, inSTZ+ Stem cell-treated group, the hearts were displaying muscle fibers and perivascular moderate deposition of the collagen fibers (figs.4C).

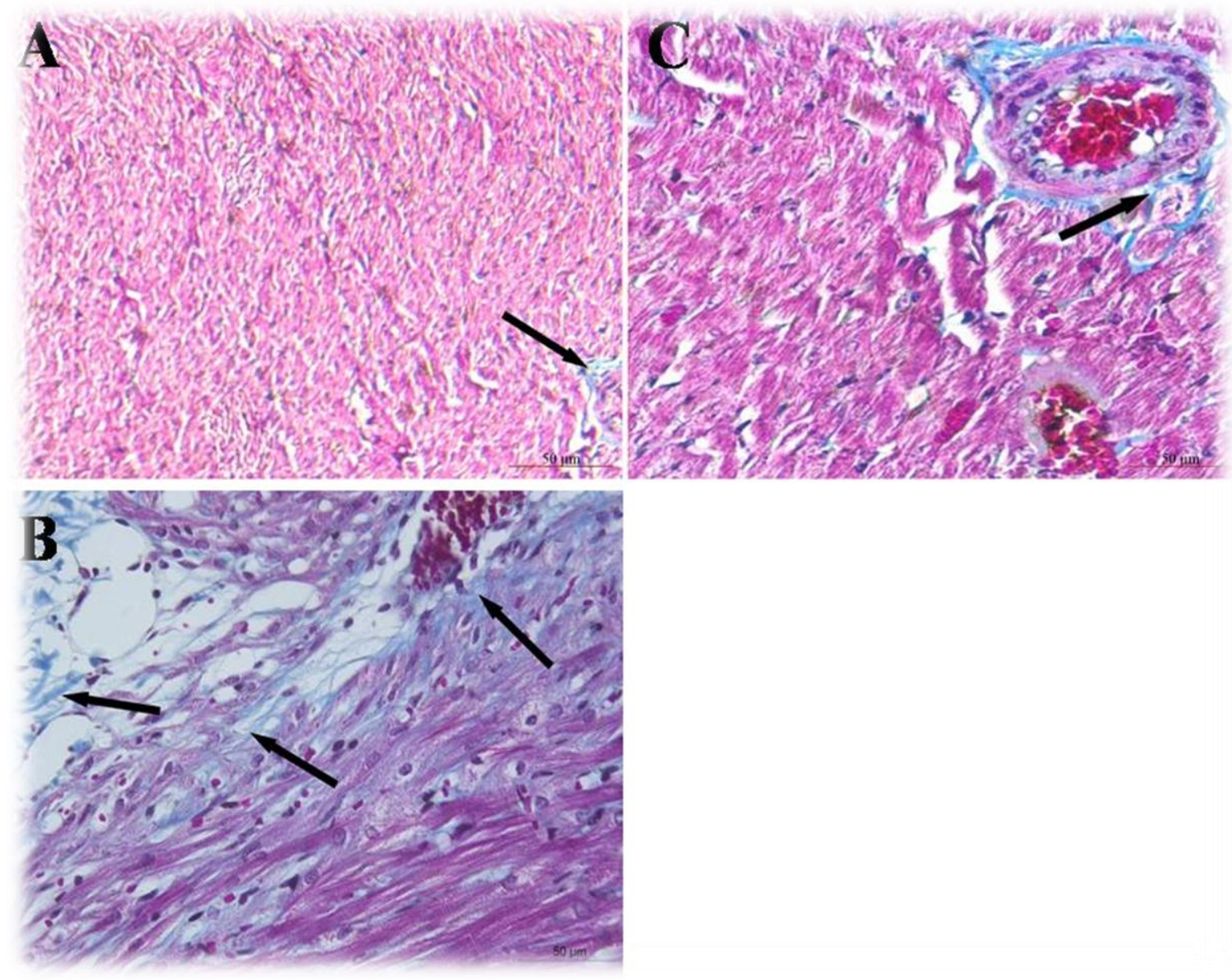


Fig. (4): A photomicrograph of masson's trichrome stained heart sections of control (A), STZ treated (B) and STZ+ stem cell treated (C) groups showing collagen fibers deposition in-between muscle fibers and around blood vessels (arrows). (A) minimal collagen fibers deposition. (B) massive collagen fibers deposition. (C) moderate deposition of the collagen fibers. (Masson's trichrome, X400)

\section{Immunohistochemical results (figs. 5):}

Immunohistochemical staining of the hearts for (eNOS) demonstrated brown coloration (positive eNOSimmunoexpression) in the vascular endothelium and the cardiomyocytes in the control group (figs.5A). However, in STZ-treated group, the heart sections showed less intensity of eNOSimmunoexpression in the vascular endothelium and the cardiomyocytes(figs.5B). While the heart sections of STZ+ Stem cell-treated group showed increased intensity of the eNOSimmunoexpression in the vascular endothelium and the cardiomyocytes(figs.5C).
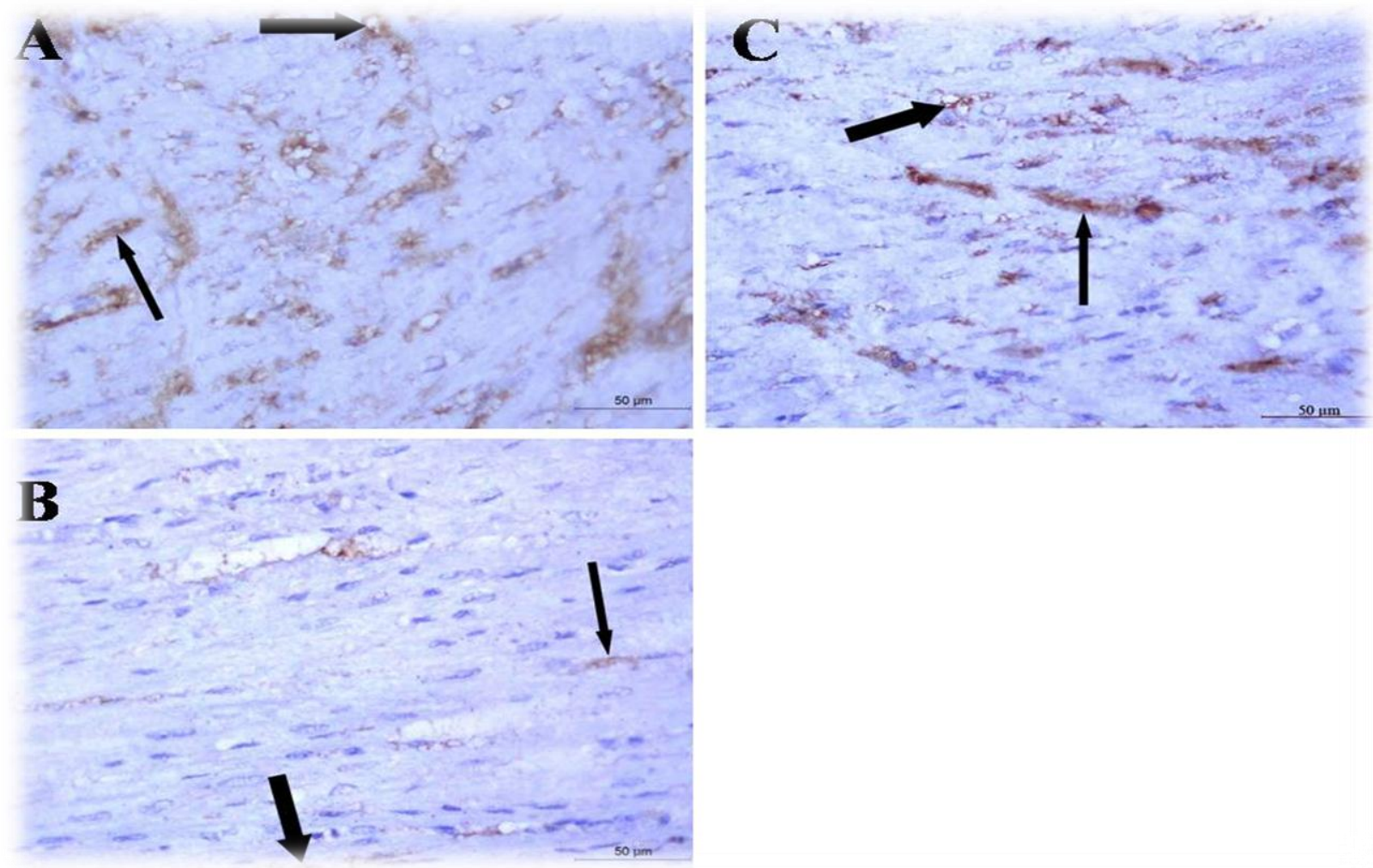

Fig. (5): A photomicrograph of eNOS immunostained heart sections of control (A), STZ treated $(B)$ and STZ+ stem cell treated $(C)$. (A) showing brown coloration (positive eNOS 
immunoexpression) in the vascular endothelium (thick arrow) and the cardiomyocytes (thin arrow). (B) showing less intensity of the eNOS immunoexpression in the vascular endothelium (thick arrow) and the cardiomyocytes (thin arrow). (C) showing increased intensity of the eNOS immunoexpression in the vascular endothelium (thick arrow) and the cardiomyocytes (thin arrow). (eNOS immunostaining, X400)

\section{Morphometric results}

The mean value of the area $\%$ of collagen fibers (figs. 6) and the intensity of eNOS immunoexpression (figs. 7) in STZ group were significantly increased as compared with the control group $(\mathrm{P}$-value $<0.05)$ (table 1$)$.

The mean value of the area \%of collagen fibers (figs. 6) and the intensity of eNOS immunoexpression (figs. 7) in STZ+stem cell group showed a significant decrease as compared with the STZ treated group $(\mathrm{P}$-value < 0.05) (table 1).

\begin{tabular}{|l|l|l|}
\hline & $\begin{array}{l}\text { Area \% of collagen } \\
\text { fibers }\end{array}$ & $\begin{array}{l}\text { Intensity of } \\
\text { immunoexpression }\end{array}$ \\
\hline Control G & $6.3 \pm 1.09$ & $35500690 \pm 1968293$ \\
\hline STZ treated G & $20.2 \pm 1.8^{*}$ & $4006886 \pm 2216594^{*}$ \\
\hline $\begin{array}{l}\text { STZ+ Stem cell- } \\
\text { treated G }\end{array}$ & $7.3 \pm 1.07$ & $27515860 \pm 2672644$ \\
\hline
\end{tabular}

*Significant $(P<0.05)$ compared with other groups.

Table (1): Comparison between all groups (control, STZ treated and STZ+ Stem celltreated G) regarding the area $\%$ of collagen fibers and the intensity of eNOS immunoexpression in heart sections (mean \pm SD) 


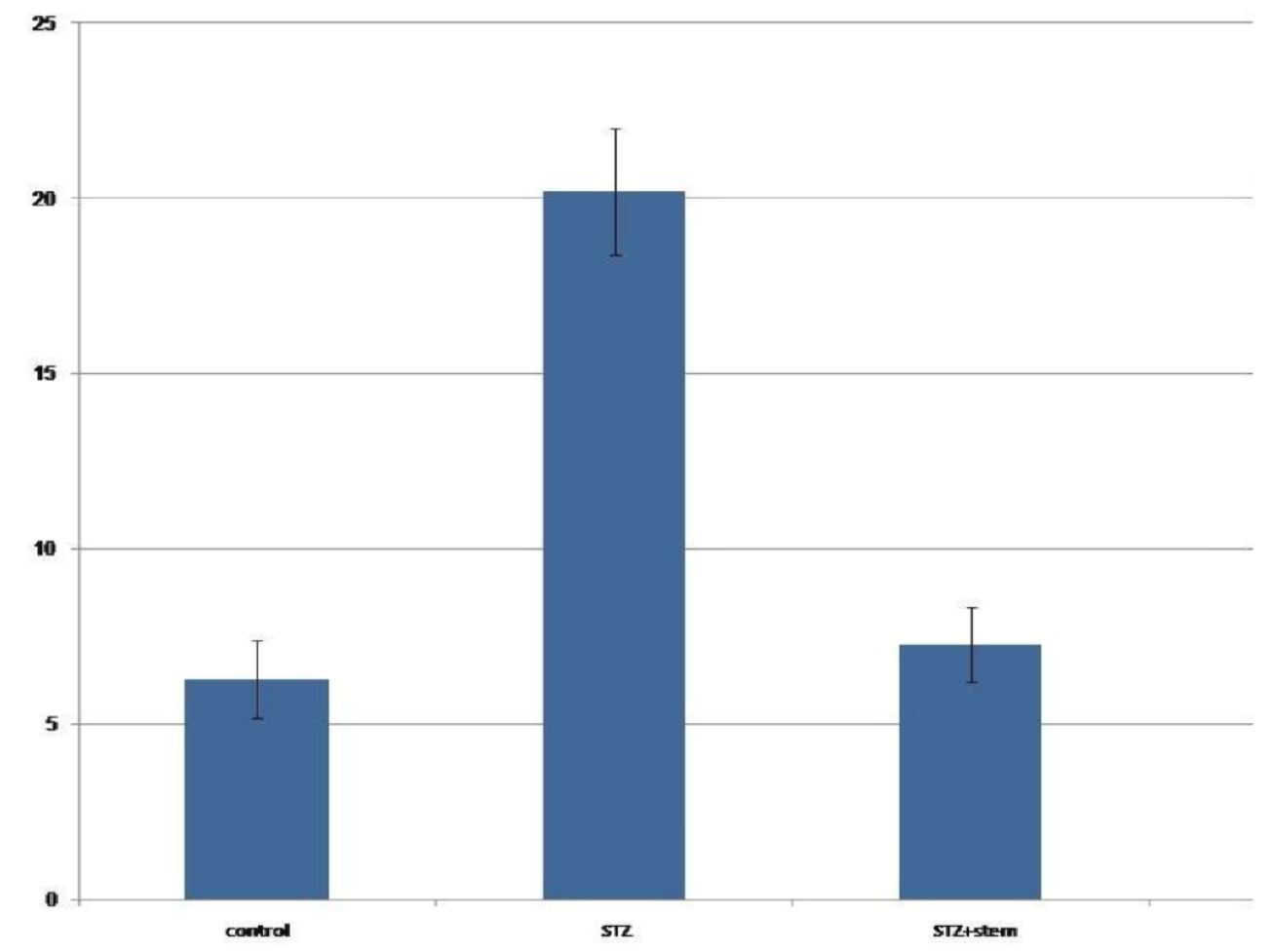

Fig. (6): Comparison between all groups (control, STZ treated and STZ+ Stem celltreated $G$ ) regarding the area \% of collagen fibers in heart sections 


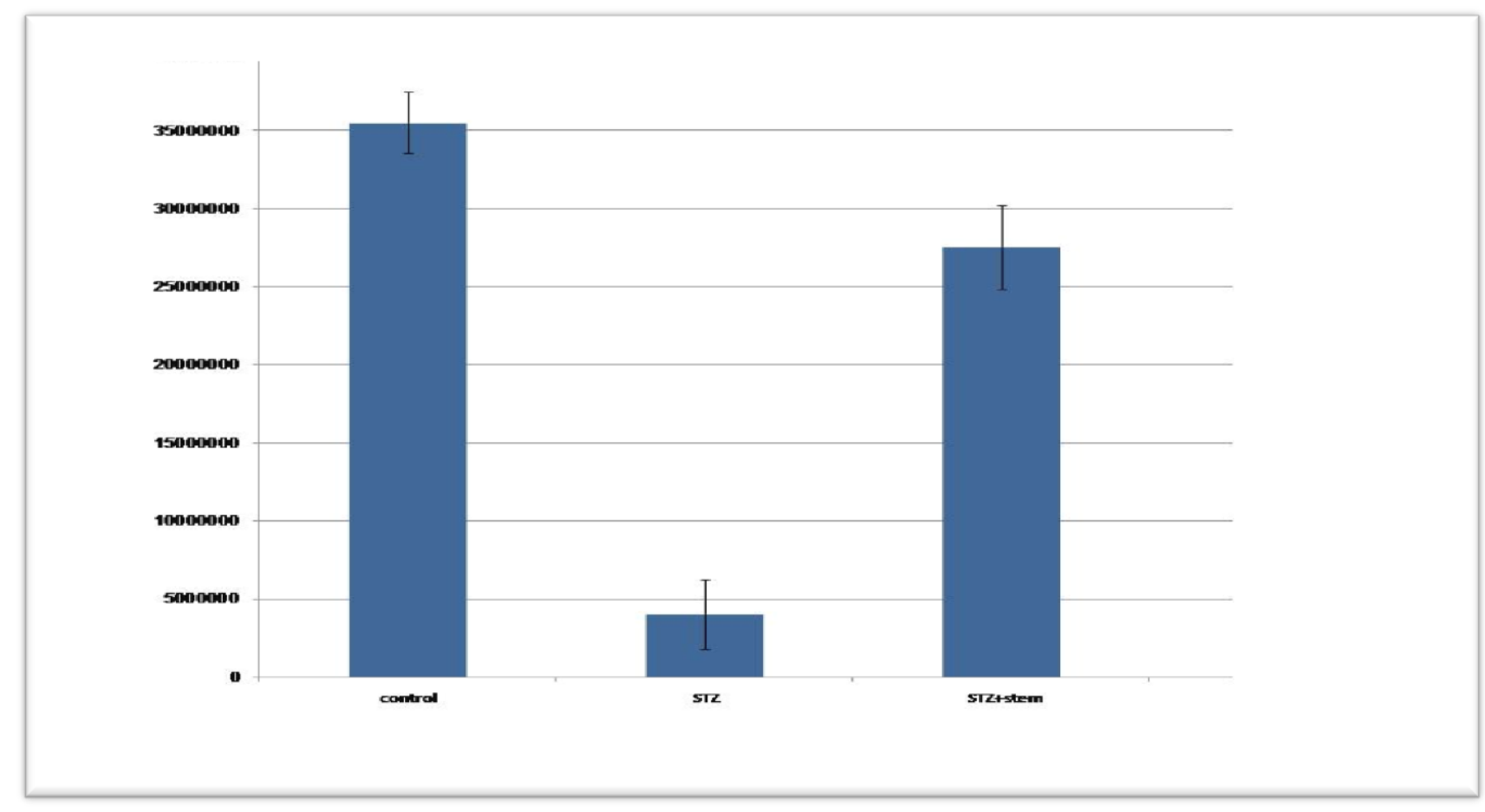

Fig. (7): Comparison between all groups (control, STZ treated and STZ+ Stem celltreated $G$ ) regarding the intensity of eNOS immunoexpression in heart sections

\section{4- Discussion:}

Diabetes mellitus (DM) is characterized by persistent dysfunction, failure and injury of several organs especially heart, kidneys, eyes, nerves and blood vessels [21].

In the current work, DM induced cardiac damage in the form of degeneration, disorganization and separation of the muscle fibers with mononuclear cellular infiltrations, congested blood vessels and extravasations. These findings were similar to other study which reported that diabetic cardiac complications included necrosis, vacuolization and hyalinization of the muscle fibers were due to inflammation and oxidative stress [22].

These results were analogous to the findings of other researchers who indicated that diabetic cardiac tissues were disordered and some of them were damaged. There was myocardial apoptosis and these pathological changes were 
due to several factors such as inflammation $[23,24]$.

In the present work, Masson's trichrome stained cardiac sections revealed significant statistical increase in the collagen fibers deposition in the walls of the blood vessels and in-between the muscle fibers in STZ treated group, in comparison with the control group. This result is in compliance with the findings of other studies that showed similar changes $[22,25]$.

Cardiac fibrosis was an outcome of oxidative stress as there were significant increase insuperoxide anion, lipid peroxidation product and proteincarbonyls levels [22]. Deposition of extracellularmatrix $(\mathrm{ECM})$ in diabetic cardiomyopathy was due to hyperglycemia that decreased endothelial cell function and microvascularblood flow [25].

Other researchers interpreted that cardiac fibrosis was due to activation of tumor growth factor-Beta (TGF- $\beta 1$ ) that caused activation of fibroblasts. This induced deposition of structural extracellular matrix proteins [24].
In the current study, light microscopic examination of the rat heart sections revealed regaining of the normal appearance of most of the cardiac muscle fibers and also revealed reduced cardiac fibrosis in STZ+ stem cell treated group. Comparable studies mentioned that pathological abnormalities including inflammation, degeneration and fibrosis were remarkably ameliorated by treatment with MSCs in diabetic rats [24].

Transplantation of stem cells in rats suffering from diabetic cardiomyopathy led to improved cardiac function of the treated animals through increased angiogenesis and attenuated cardiac remodeling. Increased matrix metalloproteinases-2(MMP-2) activity, decreased matrix metalloproteinases-9(MMP-9 ) activity and reduced collagen content were found in the diabetic myocardium after MSCs transplantation[25, 26].

In the current work, Immunohistochemical study demonstrated eNOS immunoexpression in endothelial cells and cardiomyocytes. The vascular endothelium and cardiomyocytes of diabetic hearts showed significant statistical 
decrease in the intensity of the eNOS immunoexpression, compared to the endothelium of control hearts.

These findings were analogous to the findings of other researchers who detected the importance of eNOS in healthy cardiovascular cells and suggested that a lack of eNOS in diabetes could result in changes of vascular reactivity and possibly atherosclerosis in the cardiovascular system. The authors revealed that the present findings were related to the loss of vasodilatory response occurred in diabetes [27].

Other previous studies found that the immunoexpression of eNOS decreased in the hearts of the ischemia-reperfusion diabetic rats due to oxidative stress induced by diabetes [28].

In the present work, Immunohistochemical study demonstrated increased intensity of the eNOS immunoexpression in vascular endothelium and cardiomyocytesof hearts of the STZ+stem cell treated group. In agreement, other studies found that DM resulted in impairment of the formation of
eNOS which in turn leads to decrease of vasodilatation and reduced the capillary blood flow causing diabetic cardiomyopathy. They also reported thatstem cells can be used in treatment of DMthrough direct differentiation or paracrine of trophic factors and chemokines. The main advantage of this paracrine effect is to increase proliferation and maintain the survival of cardiomyocytes and reduce the production of inflammatory response andreactive oxygen species (ROS) [25].

Nitric oxide (NO) produced by eNOS possessed antioxidant properties as it produced an increase of ferritin and heme-oxygenase-I expression that reduced superoxide anion concentrations in blood vessels [29]. The improvement in cardiac condition observed in DM after injection of MSCs can be verified by the homing of MSCs in the heart detected by the sex-determining region $\mathrm{Y}$ protein (sry gene). MSCs can prevent apoptosis through the increase of eNOS and the inhibition of myocardial fibrosis of cardiomyopathy due to decreasing the expression of matrix metalloproteinase in a rat model [30]. 


\section{5- Conclusion:}

MSCs ameliorate the pathological changes of cardiac muscle in diabetic rats. Thus stem cells provided a new line of treatment strategies in diabetic cardiomyopathy.

Conflicts of interest: There are no conflicts of interest.

\section{7- References:}

1. Peng BY, Dubey NK, Mishra VK, Tsai FC, Dubey R, Deng WP and Wei HJ : Addressing Stem Cell Therapeutic Approaches in Pathobiology of Diabetes and Its Complications.Journal of Diabetes Research, 2018; Article ID 7806435, 16P.

2. Babiker NE, Gassoum A, Abdelraheem NE, Arbab MA, ALDeaf SA, El-Sheikh MA and Musa HH. The progress of stem cells in the treatment of diabetes mellitus type 1. Prog Stem Cell, 2017; 4(1): 175188.

3. Zang L, Hao H, Liu J, Li1 Y, Han W and Mu Y.Mesenchymal stem cell therapy in type 2 diabetes mellitus. DiabetolMetabSyndr, 2017; 9:36.
4. Mousa F, Abdel-Aziz KK, Abdel Gawad H, Mahmoud SS and Elgamel MS. Bone Marrow-Derived Mesenchymal Stem cells Infusion Ameliorates Hyperglycemia, Dyslipidemia, Liver and Kidney Functions in Diabetic Rats. International Journal of Science and Research (IJSR), 2016; Volume 5 Issue 2, P: 1624-1631.

5. Graham ML, Janecek JL, Kittredge JA, Hering BJ and Schuurman HJ. The Streptozotocin-Induced Diabetic Nude Mouse Model: Differences between Animals from Different Sources: Comparative Medicine Copyright 2011 by the American Association for Laboratory Animal Science,2011; Vol 61, No 4 August 2011 P: $356-360$

6. Berardis S., Sattwika D. and Sokal E. Use of mesenchymal stem cells to treat liver fibrosis. World J Gastroenterol, 2015; 3: $742-758$.

7. Volarevic V, Nurkovic J, Arsenijevic N and Stojkovic M. Concise review: therapeutic potential of mesenchymal stem 
cells for the treatment of acute liver failure and cirrhosis. Stem Cells,2014; 32:28182823

8. Srinivasan K, Viswanad B, Asrat L, Kaul CL and Ramarao P. Combination of highfat diet-fed and low-dose streptozotocintreated rat: a model for type 2 diabetes and pharmacological screening. Pharmacol Res, 2005; 52: 313-332

9. Ngoc, K ., van Phuc,P., Nhung, T .,Thuy, D . and Nguyet,M. Improving the efficacy of type 1 diabetes therapyby transplantation of immunoisolated insulin-producing cells.Human Cell, 2011; 24: 86-95.

10. Cesaretti ML, Ginoza M, Ribeiro AB and Kohlmann OJ.Systemic hemodynamic and left ventricular function of diabetic-induced hypertensive rats. Arq Bras EndocrinolMetabol, 2010; 54(9):842-851.

11. Bluestone JA, Herold $\mathrm{K}$ and EisenbarthG.Genetics, pathogenesis and clinical interventions in type 1 diabetes. Nature,2010; 464:1293-1300.

12. Ikebukuro K, Adachi Y, Yamada Y, Fujimoto S, Seino $\mathrm{Y}$ and Oyaizu
H.Treatment of Streptozotocin-induced diabetes mellitus by transplantation of islet cells plus bone Marrow cells via portal vein in rats. Transplantation. 2002; 73(4): 512518.

13. Kajiyama H, Hamazaki TS, Tokuhara M, Masui SH, Okabayashi K, Ohnuma K, Yabe SH, Yasuda K, Ishiura SH, Okochi H and Asashima M. Pdx1-transfected adipose tissue-derived stem cells differentiate into insulin-producing cells in vivo and reduce hyperglycemia in diabetic mice. Int. J. Dev. Biol, 2010; 54, 699-705.

14. Carr CA, Stuckey DJ and Tatton L. Bone marrow-derived stromal cells home to and remain in the infarcted rat heart but fail to improve function: an in vivo cine-MRI study. Am J Physiol Heart CircPhysiol,2008; 295(2):H533.

15. Jiang TS, Cai L, Ji WY, Hui YN, Wang YS, Hu D and Zhu J. Reconstruction of the corneal epithelium with induced marrow mesenchymal stem cells in rats. Mol Vis,2010; 16: 1304-1316. 
16. Liu S., Zhao C., Yang C., Li X., Huang H., Liu N., Li S., Wang X. and Liu J. Gambogic acid suppresses pressure overload cardiac hypertrophy in rats. American Journal of Cardiovascular Disease, 2013; 3(4): 227-238

17. Kiernan J.A. Staining with dyes in one or two colours. In Histological and Histochemical Methods: Theory and Practice, 5thedition. Scion Publishing Ltd. Vantage Business Park, Bloxham Road, Banbury, UK. 2015; pp: 137-169.

18. Bancroft J.D. and Layton C. Connective and mesenchymal tissues with their stains. Theory and Practice of Histological Techniques. 7th` edition. Churchill Livingstone, Edinburgh, London, Madrid, Melbourne, New York and Tokyo, 2013; pp: 200-205.

19. Takahashi $\mathrm{T}$ and Harris RC. Role of Endothelial Nitric Oxide Synthase in Diabetic Nephropathy. Lessons from Diabetic eNOS Knockout Mice. Journal of Diabetes Research, Volume 2014; Article ID 590541.17 pages.
20. Emsley R, Dunn G and White IR. Mediation and moderation of treatment effects in randomized controlled trails of complex interventions. Stat Methods Med Res, 2010; 19:237-270.

21. American Diabetes Association. Classification and diagnosis of diabetes. Diabetes Care, 2015; (38): 8-16 and (16): $43-56$

22. Othman AI, El-Sawi MR, El-Missiry MA and Abukhalil MH. Epigallocatechin-3gallate protects against diabetic cardiomyopathy through modulating the cardiometabolic risk factors, oxidative stress, inflammation, cell death and fibrosis in streptozotocin-nicotinamide-induced diabetic rats. Biomedicine \&Pharmacotherapy,2017; 94, 362-373.

23. Ji Y, Zhao Z, Cai T, Yang $\mathrm{P}$ and Cheng $\mathrm{M}$. Liraglutide alleviates diabetic cardiomyopathy by blocking CHOPtriggered apoptosis via the inhibition of the IRE- $\alpha$ pathway. Molecular medicine reports, 2014; 9: 1254-1258 
24. Hamza AA, Fikry EM, Abdallah W and Amin A. Mechanistic insights into the augmented effect of bone marrow mesenchymal stem cells and thiazolidinediones in streptozotocinnicotinamide induced diabetic rats. Scientific Reports, 2018; 8:9827.

25. Liu M, Chen H, Jiang J, Zhang Z, Wang C, Zhang N, Dong L, Hu X, Zhu W, Yu H and Wang J. Stem cells and diabetic cardiomyopathy: from pathology to therapy. Heart Fail Rev, 2016; 21:723-736

26. Glass CE, Singal PK and Singla DK. Stem cells in the diabetic infarcted heart. Heart Fail Rev, 2010;15(6): 581-588.

27. Felaco M, Grilli A, De Lutiis MA, Patruno A, Libertini N, Taccardi AA, Napoli PD, Giulio CD, Barbacane $\mathrm{R}$ and Conti P.Endothelial Nitric Oxide Synthase (eNOS) Expression and Localization in Healthy and Diabetic Rat Hearts. Annals of Clinical \& Laboratory Science, 2001; vol. 31, no. 2, P. 179-186.

28. Xia R, Zhao B, Wu Y, Hou J, Zhang L, Xu J and Xia ZY. Ginsenoside Rb1
Preconditioning Enhances eNOS Expression and AttenuatesMyocardial Ischemia/Reperfusion Injury in Diabetic Rats. Journal of Biomedicine and Biotechnology, 2011; Article ID 767930, 8 pages.

29. Suvorava T, Nagy N, Pick S, Lieven O, Ruther U, Dao VT, Fischer JW, Weber M and Kojda G. Impact of eNOS-Dependent Oxidative Stress on Endothelial Function and Neointima Formation. Antioxidants \& Redox signaling, 2015; Volume 23.p:711723.

30. Abdel Aziz MT, El-Asmar MF, Haidara M, Atta HM, Roshdy NK, Rashed LA, Sabry D, Youssef MA, Abdel Aziz AT and Moustafa M. Effect of bone marrowderived mesenchymal stem cells on cardiovascular complications in diabetic rats: Med SciMonit, 2008; 14(11): BR 249255. 